Albrecht Reuß

\title{
Mit Nachhaltigkeitsindikatoren die Flächennutzungsplanung steuern
}

Eine indikatorenbasierte Nachhaltigkeitsprïfung könnte ein Instrument zur Umsetzung der Leitvorstellung „nachhaltige Raumentwicklung“ sein

\section{Using Sustainability Indicators to Control Zoning Plans}

A sustainability assessment that is based on indicators can be an instrument to implement the paradigm of "sustainable spatial development"

\section{Kurzfassung}

Der Umgang mit der gesetzlich vorgeschriebenen Leitvorstellung einer nachhaltigen Raumentwicklung in der Planung ist durch eine große Unsicherheit bestimmt. Um dieser zu begegnen, wird hier das Konzept einer indikatorenbasierten Nachhaltigkeitsprüfung für die Flächennutzungsplanung vorgestellt. Diese Prüfung unterstützt die Abwägung, indem die Leitvorstellung einer nachhaltigen Raumentwicklung konkretisiert und überprüfbar gemacht wird. Als Bewertungskriterien für die Prüfung dienen bekannte Nachhaltigkeitsindikatoren. Sie erhalten durch die Nachhaltigkeitsprüfung eine stärkere Planungs- und Steuerungsfunktion. In diesem Beitrag werden eine Methodik für eine indikatorenbasierte Nachhaltigkeitsprüfung vorgeschlagen und Erfolgsfaktoren für die Integration der Nachhaltigkeitsziele in die Planung benannt.

\begin{abstract}
Dealing with the paradigm of "sustainable spatial development" (in German planning) is still characterised by some uncertainties. In order to respond to these uncertainties, the following article presents the concept of an indicator - based sustainability assessment. It assists the consideration of zoning plans by putting the paradigm of "sustainable spatial development" in concrete terms and making it checkable. The criteria for the assessment are familiar sustainability indicators. As a result of the sustainability assessment, the planning and control functions of these criteria increase. This article proposes a methodology for indicator-based sustainability assessment and names the success factors for the integration of sustainability goals into planning.
\end{abstract}

\section{Notwendigkeit einer indikatorenbasierten Nachhaltigkeitsprüfung}

Aus der gesetzlich verankerten Leitvorstellung einer nachhaltigen Raumentwicklung ( $\$ 1$ Abs. 2 ROG; $\$ 1$ Abs. 5 BauGB) ergibt sich die Notwendigkeit, diese zu operationalisieren. Zahlreiche Kommunen haben zu diesem Zweck Nachhaltigkeitsindikatoren aufgestellt, die eine wichtige Funktion in der Kommunikation mit den Bürgern und der Berichterstattung innerhalb der
Verwaltung übernehmen, aber bislang kaum als Planungs- und Steuerungsinstrumente eingesetzt werden (Gehrlein 2002, S. 243). Offenbar lassen Indikatoren sich noch nicht ausreichend in Planungsverfahren integrieren, was notwendig wäre, um die Abwägung zu unterstützen. 
Im Zusammenhang mit Umweltverträglichkeitsprüfungen wird ebenfalls über die Operationalisierung der Leitvorstellung einer nachhaltigen Raumentwicklung diskutiert und dabei eine Erweiterung dieser Prüfungen zu Nachhaltigkeitsprüfungen vorgeschlagen (z.B. Jacoby 2000, S. 532). Bei diesem Ansatz stellt nicht die Integration der Prüfungen in die Planungsverfahren das Problem dar, sondern die Operationalisierung der Leitvorstellung Nachhaltigkeit. Sie ist in der Methodik der Umweltverträglichkeitsprüfungen bislang noch nicht gelungen.

Die indikatorenbasierte Nachhaltigkeitsprüfung kombiniert nun die Stärken dieser beiden Ansätze, indem sie die vorhandenen Nachhaltigkeitsindikatoren als Grundlage für eine Prüfung heranzieht und in ein Verfahren integriert, das auf den Erfahrungen der Umweltprüfungen beruht. Das Grundkonzept dieser Prüfung sieht vor, den Einfluss des Plans auf die Entwicklung der Indikatoren und somit seinen Beitrag zu einer nachhaltigen Raumentwicklung zu analysieren. Durch eine solche Prüfung kann der Informationsstand innerhalb eines Planungsverfahrens erhöht und das Ergebnis der Abwägung verbessert werden. Weitere Vorteile sind die hohe Anschaulichkeit und Transparenz innerhalb des Planungsverfahrens, wodurch in Anlehnung an kooperative Planungsverfahren (vgl. Bischoff et al. 2001) die Akzeptanz der Planungen gesteigert und die Umsetzung erleichtert wird.

Als Anwendungsgebiet der indikatorenbasierten Nachhaltigkeitsprüfung wird bislang vor allem die Ebene der Flächennutzungsplanung diskutiert.

\section{Ausgangslage}

Als Grundlage benötigt die indikatorenbasierte Nachhaltigkeitsprüfung einen erprobten Indikatorensatz, für den bereits Daten aus den vergangenen Jahren vorliegen. Diesen liefert das ExWoSt-Programm „Städte der Zukunft" des BBR (Fuhrich 2001). Die Indikatoren des Forschungsfeldes sind in Abbildung 1 dargestellt. Mittlerweile ist der Indikatorensatz etwas modifiziert worden (vgl. Internet: www.staedte-der-zukunft.de). Es muss in dieser Betrachtung allerdings auf den Stand zurückgegriffen werden, für den in den vergangenen Jahren Daten erhoben wurden.

Im Zuge des Forschungsfeldes sollten die erarbeiteten Indikatoren helfen, die Wirkung von Nachhaltigkeitsstrategien in vier Modellstädten zu überprüfen. Zu diesem Zweck erstellten die Städte Heidelberg, Münster, Dessau und Güstrow regelmäßige Indikatorenberichte. Die indikatorenbasierte Nachhaltigkeitsprüfung soll den bisherigen Ansatz des Forschungsfeldes ergänzen und Möglichkeiten aufzeigen, wie die Indika-
Abbildung 1

Die ExWoSt-Indikatoren des Forschungsfeldes „Städte der Zukunft"

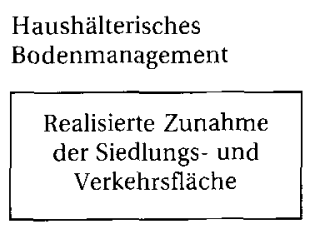

Stadtverträgliche

Mobilitätssteuerung
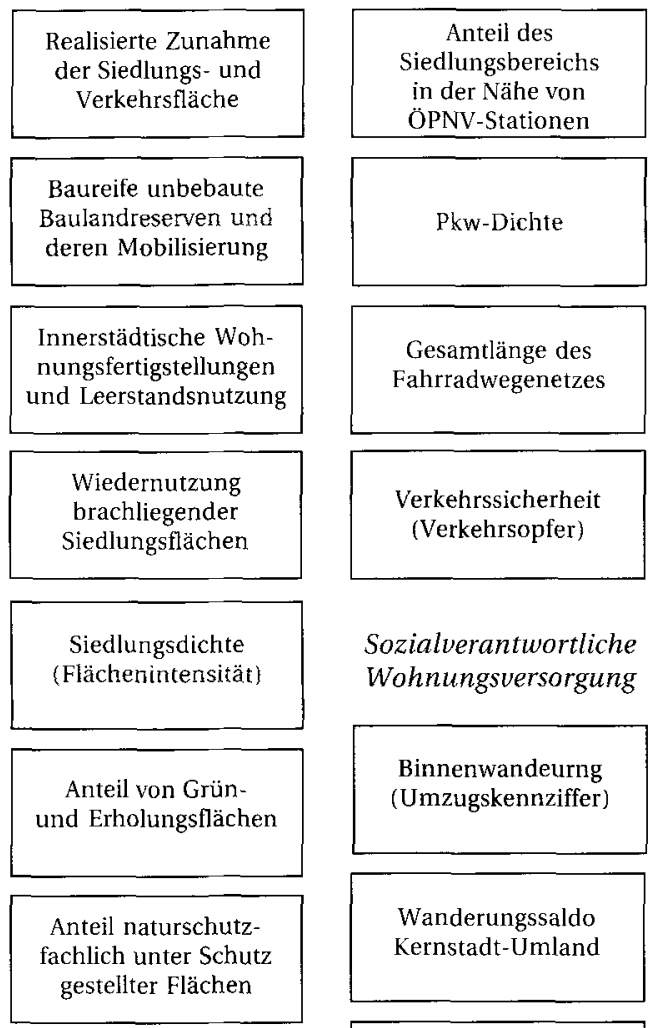

Vorsorgender

Umweltschutz

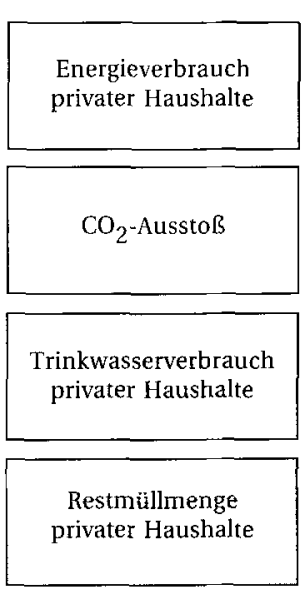

grau unterlegt:

Indikatoren, die durch den Flächennutzungsplan nicht beeinflussbar sind

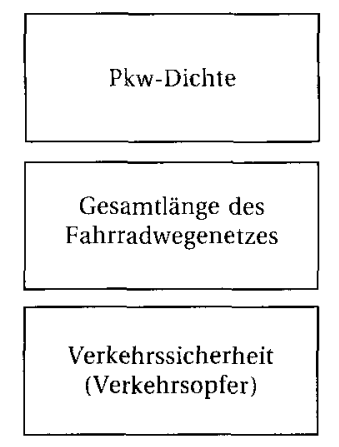

Sozialverantwortliche Wohnungsversorgung
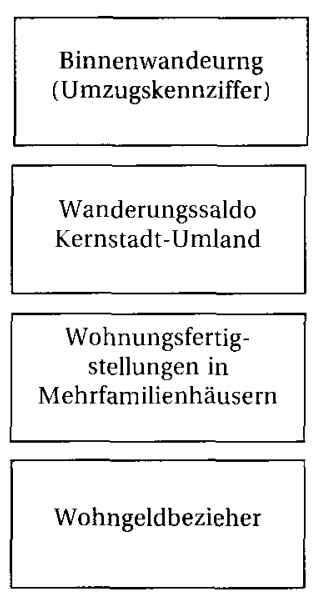

Standortsichernde Wirtschaftsförderung
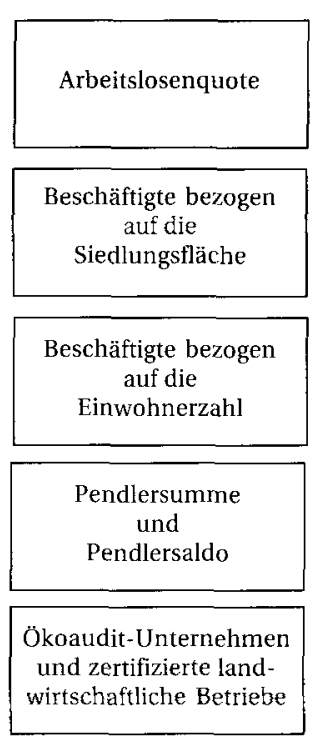
toren einen stärker steuernden Charakter erhalten können. Am Beispiel Heidelberg wurde dies einmal durchgespielt (Reuß 2002), da für diese Stadt sowohl Daten zu den betrachteten Indikatoren als auch aktuelle Planungen auf der Flächennutzungsebene (Modell Räumliche Ordnung, Heidelberg 2000) vorlagen.

Dieser Beitrag widmet sich nun der Methodik der indikatorenbasierten Nachhaltigkeitsprüfung und versteht sich zunächst als Vorschlag für die praktische Anwendung in Heidelberg und anderen Städten. Zur besseren Anschaulichkeit wird die Beschreibung der Methodik anhand zweier Beispielindikatoren vorgenommen: dem Indikator "Siedlungs- und Verkehrsfläche" und dem Indikator „Umlandwanderung“. Die Darstellung der Ergebnisse bezieht dann allerdings weitere Indikatoren mit ein.

\section{Methodik}

Die indikatorenbasierte Nachhaltigkeitsprüfung besteht aus drei Schritten. Zunächst wird ein Wirkungsmodell erstellt, in dem jedem Indikator des gewählten Indikatorensatzes ein oder mehrere Prüfkriterien zugewiesen werden. Unter einem Prüfkriterium wird hier eine Messgröße verstanden, die räumlich darstellbar ist und unmittelbar dem Planentwurf entnommen werden kann. Der Umweg über die Prüfkriterien ist notwendig, da bestehende Nachhaltigkeitsindikatoren in der Regel allgemeine Messgrößen darstellen, die nicht direkt auf den Flächennutzungsplan zugeschnitten sind. Die Beziehung zwischen Prüfkriterium und Indikator hat dabei den Charakter einer Wirkungshypothese. Im zweiten Schritt werden die Prüfkriterien anhand des Planentwurfs gemessen und in Bezug zu den Werten des Ist-Zustandes gesetzt. Es können nun im dritten Schritt Schlüsse auf die Entwicklung des Ausgangsindikators gezogen werden. Dadurch wird eine Bewertung über den Beitrag des Plans zu einer nachhaltigen Raumentwicklung möglich. Es ist hingegen nicht das Ziel, die reale Entwicklung der Indikatoren zu prognostizieren. Diese wird neben dem Flächennutzungsplan durch zu viele anderen Einflüsse bestimmt.

\subsection{Das Wirkungsmodell}

Ausgehend von dem ExWoSt-Indikatorensatz werden jedem Indikator ein oder mehrere Prüfkriterien zugewiesen. Dabei sind folgende Fälle zu unterscheiden:

\section{(1) Direkter Wirkungszusammenhang} zwischen Prüfkriterium und Indikator

Hierbei wird der Indikator direkt durch den Flächennutzungsplan beeinflusst. Dies ist vor allem für flächenhafte Indikatoren der Fall. Die Wirkung des Prüfkriteriums auf den Plan ist in diesem Fall problemlos zu ermitteln. Im vorliegenden Beispiel trifft dies für den Indikator "Siedlungs- und Verkehrsfläche“ zu.

\section{(2) Indirekter Wirkungszusammenhang zwischen Prüfkriterium und Indikator}

Dies ist für Indikatoren der Fall, die nicht flächenhaft sind und daher nicht direkt durch den Flächennutzungsplan beeinflusst werden. Der Zusammenhang zwischen Prüfkriterium und Indikator ist dabei nur schwer zu quantifizieren. Durch die Betrachtung der Entwicklung des Prüfkriteriums lässt sich allerdings näherungsweise eine Tendenz für die Entwicklung des Indikators ableiten. Für eine Bewertung des Planentwurfs reicht dies meist aus. Bei indirekten Wirkungszusammenhängen kann ein Indikator gleichzeitig durch mehrere Prüfkriterien beeinflusst werden. Der Indikator „Umlandwanderung“ ist ein Beispiel mit indirektem Wirkungszusammenhang.

\section{(3) Indirekter Wirkungszusammenhang zwischen Indikatoren}

Da innerhalb eines Indikatorensatzes Wechselwirkungen auftreten können, müssen im Wirkungsmodell auch Wirkungen eines Indikators auf andere Indikatoren mitbetrachtet werden. So hat etwa der Indikator „Umlandwanderung“ einen Einfluss auf den Indikator „Ein-/Auspendler“, da Fortzieher zu einem großen Anteil ihre Arbeitsplätze behalten und dadurch Einpendler werden (Geier et al. 2001, S. 23). Prüfkriterien, die den Indikator „Umlandwanderung“ betreffen, betreffen also ebenso den Indikator „Ein-/Auspendler“.

\section{(4) Kein Wirkungszusammenhang}

Für einige Indikatoren lassen sich keine Prüfkriterien zuweisen, da sie durch den Flächennutzungsplan gänzlich unbeeinflusst bleiben. Diese Indikatoren scheiden in der indikatorenbasierten Nachhaltigkeitsprüfung aus der weiteren Betrachtung aus. Sie sind in Abbildung 1 grau unterlegt.

Es ist zu empfehlen, für die Erstellung des Wirkungsmodells das Wissen mehrerer Fachleute hinzuzuziehen, denn das Wirkungsmodell hat eine entscheidende Bedeutung für die spätere Bewertung, und aufgrund des integrativen Charakters der Leitvorstellung der nachhaltigen Raumentwicklung sind praktisch alle Bereiche der Raumentwicklung betroffen. Daher wurde 
für die beispielhafte Untersuchung des Heidelberger Modells Räumliche Ordnung eine Expertenbefragung durchgeführt. Die zehn befragten Expertinnen und Experten arbeiten in Wissenschaft und Praxis zu den Themen Flächennutzungsplanung, Umweltverträglichkeitsprüfung, nachhaltige Raumentwicklung und Indikatorenforschung.

Der Indikator "Siedlungs- und Verkehrsfläche“ unterlag dabei nur wenigen Diskussionen, da er sich direkt durch das Prüfkriterium "Geplante Siedlungs- und Verkehrsfläche" abbilden lässt (siehe Abb. 2). Einige Experten äußerten Skepsis hinsichtlich der tatsächlichen Umsetzung des Flächennutzungsplans. Es ist allerdings eine Grundannahme der indikatorenbasierten Nachhaltigkeitsprüfung, dass der Plan in der dargestellten Weise realisiert werden soll. Ohne diese Grundannahme entzöge sich der Plan jeglicher Bewertung.

\section{Abbildung 2}

Das Wirkungsmodell für den Indikator

"Siedlungs- und Verkehrsfläche“

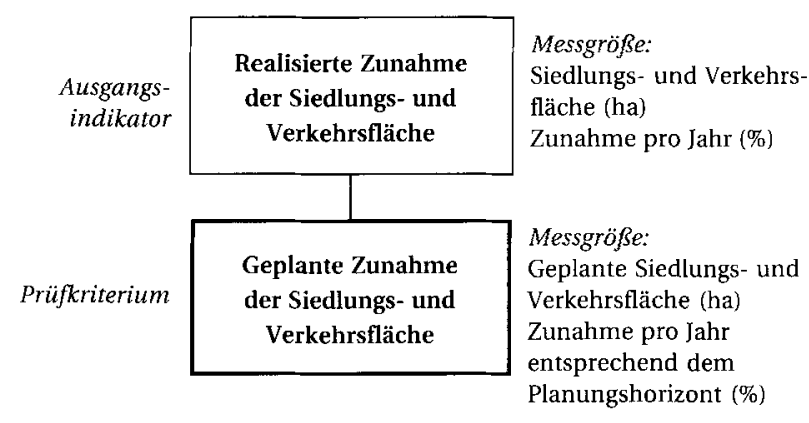

Quelle: eigene Darstellung

Weniger einheitlich war die Analyse des Indikators „Umlandwanderung“. Es stellte sich zunächst die Frage, ob dieser Indikator überhaupt durch den Flächennutzungsplan beeinflussbar ist. Nur eine knappe Minderheit der Experten war dieser Auffassung und sprach sich für das Prüfkriterium „Erreichbarkeit von Grünund Freiflächen" zur Abbildung des Indikators aus. Begründet wurde dies damit, dass nach mehreren Studien der Wunsch nach Wohnen im Grünen den bedeutendsten Fortzugsgrund darstellt (Stadt Dortmund 2001, S. 11). Obwohl eine knappe Mehrheit der Experten den Indikator als unbeeinflussbar ansah, wurde dieses Prüfkriterium aufgenommen. In der Diskussion vor Ort ist die Umlandwanderung ein so zentrales Thema, dass dies die Betrachtung des Indikators rechtfertigt, selbst wenn der Wirkungszusammenhang zwischen Grünerreichbarkeit und Umlandwanderung relativ unsicher ist. Durch die Auswahl des Prüfkriteriums „Grünerreichbarkeit“ wird gleichzeitig einer anderen möglichen Argumentationsweise widerspro- chen, die in der Ausweisung neuer Bauflächen für Einund Zweifamilienhäuser ein Werkzeug gegen die steigende Umlandwanderung sieht. In Bezug auf die Grünversorgung ist dieses Vorgehen kontraproduktiv und dürfte für sich allein genommen die Umlandwanderung kaum abdämpfen.

Mit der Abbildbarkeit des Indikators „Umlandwanderung“ durch das Prüfkriterium „Grünerreichbarkeit“ wird nun auch der Indikator „Pendlerströme“ abbildbar, da ein Wirkungszusammenhang zwischen diesen beiden Indikatoren besteht. Der Ausschnitt des Wirkungsmodells für diese Indikatoren ist in Abbildung 3 dargestellt. Die im Wirkungsmodell aufgeführten Prüfkriterien sind die Grundlage für die weitere Bewertung.

\section{Abbildung 3}

Das Wirkungsmodell für den Indikator „Umlandwanderung“

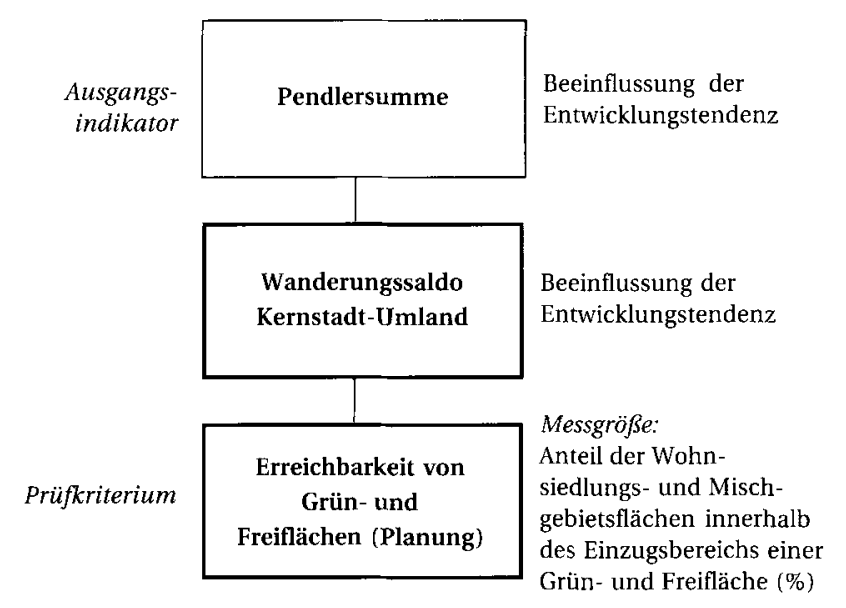

Quelle: eigene Darstellung

\subsection{Messung der Prüfkriterien}

Die Messung der Prüfkriterien nimmt zeitlich einen vergleichsweise geringen Umfang ein, da per definitionem nur Messgrößen Anwendung finden, die unmittelbar aus dem Planentwurf entnommen werden können. Voraussetzung dafür ist, dass die Darstellungen des Planentwurfs in digitalisierter Form vorliegen. Andernfalls sind zunächst aufwändige Digitalisierungen erforderlich.

Das Prüfkriterium „Geplante Siedlungs- und Verkehrsfläche“ bezieht sich auf eine Größe, die ohnehin im Laufe des Planungsprozesses eine zentrale Rolle spielt und daher ohne Aufwand zu erheben ist. Mehr noch: In der Regel stellt sie über die vorgelagerten Bedarfsrechnungen den "Inputfaktor“ für die Planung dar und keinen „Outputfaktor“. Die Bedeutung der Nachhaltigkeitsprüfung für dieses Prüfkriterium liegt daher nicht 
in der reinen Informationsgewinnung, sondern verstärkt darin, die Werte transparent zu machen und in Bezug zur bisherigen Entwicklung zu setzen (siehe Abschnitt 3.3). Im Heidelberger Beispiel wird nach dem Modell Räumliche Ordnung die Zunahme der Siedlungs- und Verkehrsfläche bis zum Jahr 2015 unverändert durchschnittlich jährlich $0,3 \%$ betragen.

Für das Prüfkriterium „Erreichbarkeit von Grün- und Freiflächen" ist eine Konkretisierung der Messgröße durch eine einfache Modellierung erforderlich. Im vorliegenden Fall wurden als Mindestgröße einer Grünfläche 2 ha und als unmittelbarer Einzugsbereich der Grün- und Freiflächen eine Entfernung von $300 \mathrm{~m}$ angenommen. Diese sehr geringe Entfernung, die einer Gehzeit von etwa fünf Minuten entspricht, wurde gewählt, um den Wunsch der Menschen nach „Wohnen im Grünen" treffsicher abzubilden. Nun wurde sowohl für den Ist-Zustand als auch für die Planung der Anteil der Wohn- und Mischgebietsfläche ermittelt, der innerhalb dieses Einzugsbereichs liegt (siehe Abb. 4).

\section{Abbildung 4}

Erholungswert der Wohn- und Mischgebietsflächen in Heidelberg

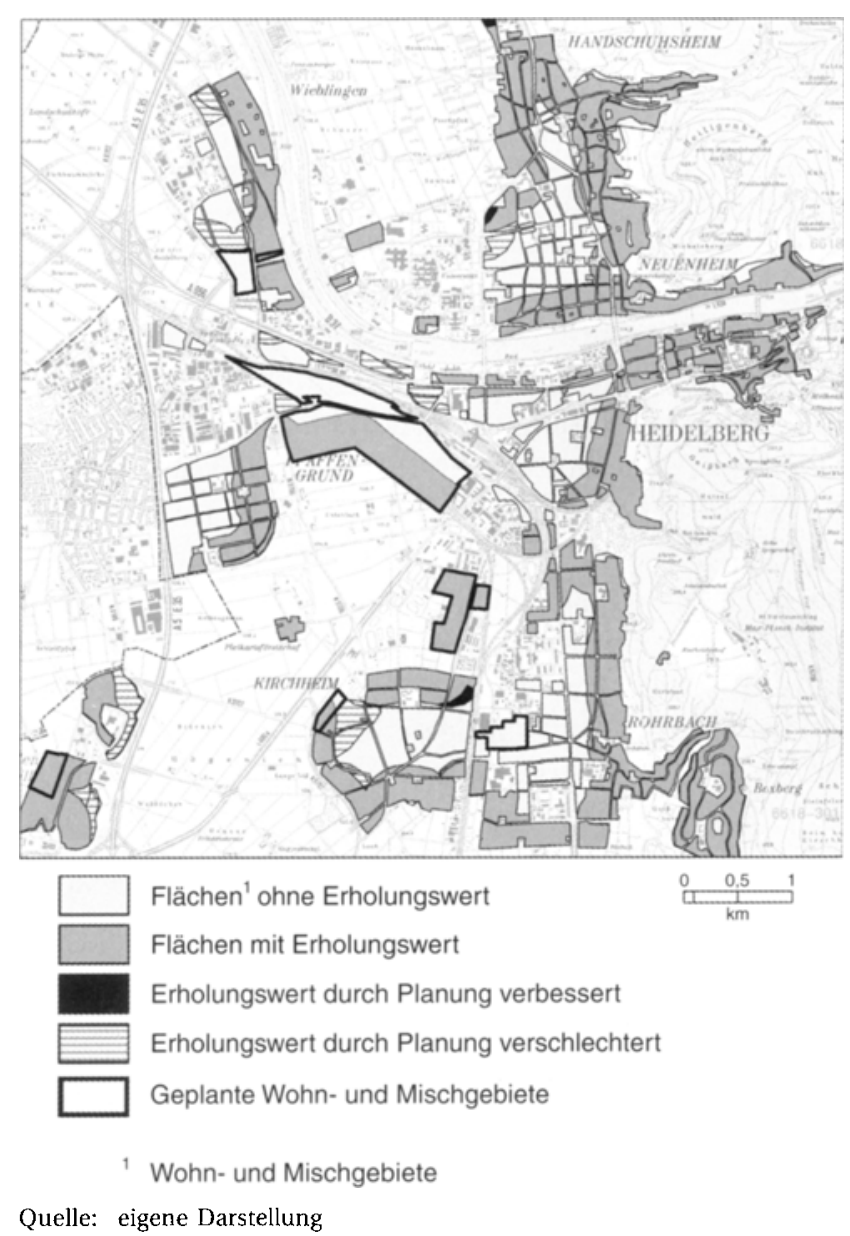

Unberücksichtigt bleiben dabei die Einwohnerdichte in den betrachteten Wohn- und Mischgebieten, die Erholungsqualität der Grün- und Freiflächen sowie die realen Wegelängen. Unter dem Aspekt des Erhebungsaufwands und der Anschaulichkeit ist auf dieser eher groben Planungsstufe diese Art der Modellierung ausreichend. Für Heidelberg stellt man fest, dass der Anteil der Wohn- und Mischgebiete mit Grünversorgung durch die Planrealisierung von 74,3 auf $70,8 \%$ sinkt.

\subsection{Interpretation und Bewertung}

Zur Interpretation der gemessenen Prüfkriterien betrachtet man diese nun in Zeitreihen. Für Prüfkriterien, die in einem direkten Wirkungszusammenhang zum Ausgangsindikator stehen, ist die Zeitreihe des Prüfkriteriums identisch mit einer Fortschreibung der Zeitreihe des Indikators. Für Prüfkriterien mit einem indirekten Wirkungszusammenhang zum Ausgangsindikator muss das Prüfkriterium in einer eigenen Zeitreihe analysiert werden.

Die Zeitreihe für den Indikator und das Prüfkriterium "Siedlungs- und Verkehrsfläche" ist in Abbildung 5 dargestellt. Man erkennt, dass der relativ stabile Trend im Flächenverbrauch seit 1981 anhält, wenn die Flächenpotenziale des Modells Räumliche Ordnung voll ausgeschöpft werden. Es ist damit festzuhalten, dass der Indikator "Siedlungs- und Verkehrsfläche" nicht in die gewünschte Richtung gelenkt wird und der Plan unter diesem einen Gesichtspunkt nicht zu einer nachhaltigen Raumentwicklung beiträgt.

Die Analyse des Indikators "Umlandwanderung“ mit Hilfe der Messwerte für das Prüfkriterium „Grünerreichbarkeit" ergibt, dass aufgrund der abnehmenden Grünversorgung die Umlandwanderung durch das Modell Räumliche Ordnung nicht gedämpft werden kann. Auch unter diesem Aspekt unterstützt die Planung das Ziel einer nachhaltigen Raumentwicklung

Abbildung 5

Zeitreihe für den Indikator "Siedlungs- und Verkehrsfläche“ in Heidelberg

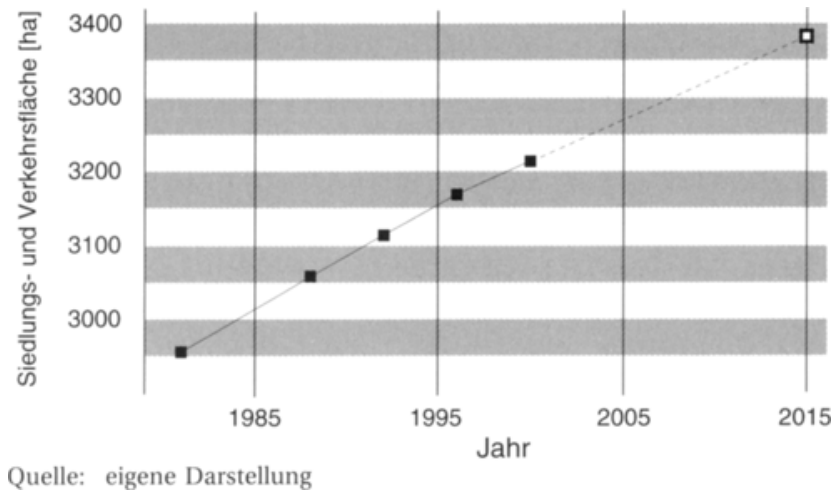


nicht. Allerdings kann für dieses Prüfkriterium die Aussage nicht durch eine Zeitreihenbetrachtung gestützt werden, da keine Daten über die Zeit vor 1999 vorliegen. Setzt man nun die Tendenz der weiterhin zunehmenden Umlandwanderung in das Wirkungsmodell ein, so erhält man auch für den Indikator „Pendlersum: me" eine steigende Tendenz. Damit werden durch das Prüfkriterium „Grünversorgung“ insgesamt zwei Indikatoren nicht in die gewünschte Richtung beeinflusst.

\subsection{Zusammenschau der Indikatoren}

Die Methodik der indikatorenbasierten Nachhaltigkeitsprüfung wurde hier anhand von zwei Beispielindikatoren dargestellt. Um zu einer Gesamtaussage über den Beitrag des Plans zu einer nachhaltigen Raumentwickiung zu kommen, muss der Indikatorensatz in seiner Gesamtheit betrachtet werden. Für Heidelberg lässt sich dabei zusammenfassend festhalten, dass insgesamt 16 der 24 ExWoSt-Indikatoren durch das Modell Räumliche Ordnung direkt oder indirekt beeinflusst werden. Für diese 16 Indikatoren ließen sich zehn Prüfkriterien ableiten. Aus den Messergebnissen wurde gefolgert, dass von den 16 AusgangsIndikatoren nur die Indikatoren ,Innerstädtische Wohnungsfertigstellungen", „Gesamtlänge des Fahrradwegenetzes" und „Anteil von Grün- und Erholungsflächen" in die gewünschte Richtung beeinflusst werden (siehe Abb. 6).

Eine weitergehende Aggregation der Indikatoren für die Gesamtbewertung ist nicht vorgesehen, da der Indikatorensatz bereits eine gleichrangige, anschauliche und nachvollziehbare Auswahl bedeutsamer MessgröBen darstellt. Es muss den politischen Entscheidungsträgern und der Öffentlichkeit überlassen bleiben, welche Schlüsse aus der Gesamtschau der Indikatoren zu ziehen sind.

Auf die Schlüsselfunktion des Indikators „Siedlungsund Verkehrsfläche“ soll jedoch hingewiesen werden. Dieser Indikator ist wie kein anderer direkt mit der Flächennutzungsplanung verknüpft. Zugleich wirkt er indirekt auf eine große Zahl anderer Indikatoren ein. Mit zunehmender Siedlungsfläche sinkt bei stagnierender Einwohnerzahl die Siedlungsdichte, und diese wiederum beeinflusst weitere Indikatoren wie " $\mathrm{CO}_{2}$ Ausstoß“, „Pkw-Dichte“ und „Energieverbrauch privater Haushalte". Insgesamt sind im vorliegenden Fall bis zu zehn der 16 Ausgangsindikatoren durch weitere Flächenausweisungen betroffen. Diese Schlüsselfunktion der Siedlungsfläche lässt sich ungeachtet der einzelnen politischen Schlüsse zweifelsfrei festhalten. Nur wenn es gelingt, den Flächenverbrauch entscheidend zu verringern, wird das Modell Räumliche Ordnung den Zielen einer nachhaltigen Raumentwicklung ent- sprechen können (zur Schlüsselfunktion der Siedlungsfläche siehe auch Birkmann 1999, S. 127; Apel et al. 2001, S. 42).

\subsection{Die Betrachtung von Alternativen}

Besonders geeignet ist die indikatorenbasierte Nachhaltigkeitsprüfung zur vergleichenden Bewertung von Planalternativen. In vergleichenden Betrachtungen gewinnen die indirekten Wirkungszusammenhänge eine höhere Aussagekraft, da sie zwar keine quantifizierbaren Prognosen zulassen, aber doch die Einstufung mehrerer Alternativen in Rangfolgen nach dem Prinzip „besser als“ oder "schlechter als“. Im oben dargestellten Beispiel wurden die Alternativen „Planung“ und „Ist-Zustand“ betrachtet. Doch die Methodik bietet sich insbesondere auch für die Betrachtung von Planungsalternativen an.

Im Heidelberger Fall wurde diese Alternativenbetrachtung für das geplante Gewerbegebiet "Marienhof" durchgeführt, welches innerhalb von Rat und Verwaltung umstritten ist (Stadt Heidelberg 2000, S. V). Es hat eine Fläche von etwa 110 ha und liegt unmittelbar südwestlich des Autobahnkreuzes Heidelberg. Als Alternativen wurden die Ausweisung dieser Fläche als Gewerbegebiet und die Belassung der Fläche als landwirtschaftliche Fläche untersucht.

Im Ergebnis schneidet die Alternative „landwirtschaftliche Fläche" in vier von acht betroffenen Prüfkriterien gleich gut und in den anderen vier besser ab als die Alternative "Gewerbegebiet“. In der Folge werden in der Alternative "landwirtschaftliche Fläche" nun sechs statt drei Indikatoren in die gewünschte Richtung beeinflusst. Dies liegt in dem nun deutlich zurückgehenden Flächenverbrauch begründet. Der Zuwachs der Siedlungsfläche betrüge bis 2015 statt $0,3 \%$ nur noch $0,2 \%$ jährlich. Ein positiver Effekt auch für die Siedlungsdichte ist allerdings dadurch nicht festzustellen, da der Flächenverbrauch insgesamt noch immer zunimmt. Eine leichte Verbesserung erfährt das Prüfkriterium „ÖPNV-Versorgung“, da das Gewerbegebiet "Marienhof" beim bisherigen Stand der Planung kaum durch den ÖPNV erschlossen wäre.

Das Ergebnis der Alternativenbetrachtung ist die Handlungsempfehlung, auf die Ausweisung der Fläche als Gewerbegebiet zu verzichten. Einschränkend muss hinzugefügt werden, dass es bislang noch unzureichend gelungen ist, die möglichen positiven Effekte einer Gewerbeausweisung in das Bewertungssystem mit aufzunehmen. Den befragten Expertinnen und Experten erschien etwa der Zusammenhang zwischen Flächenausweisungen und der Schaffung von Arbeitsplätzen als zu vage. 
Abbildung 6

Beeinflussung der Nachhaltigkeitsindikatoren durch das Modell Räumliche Ordnung

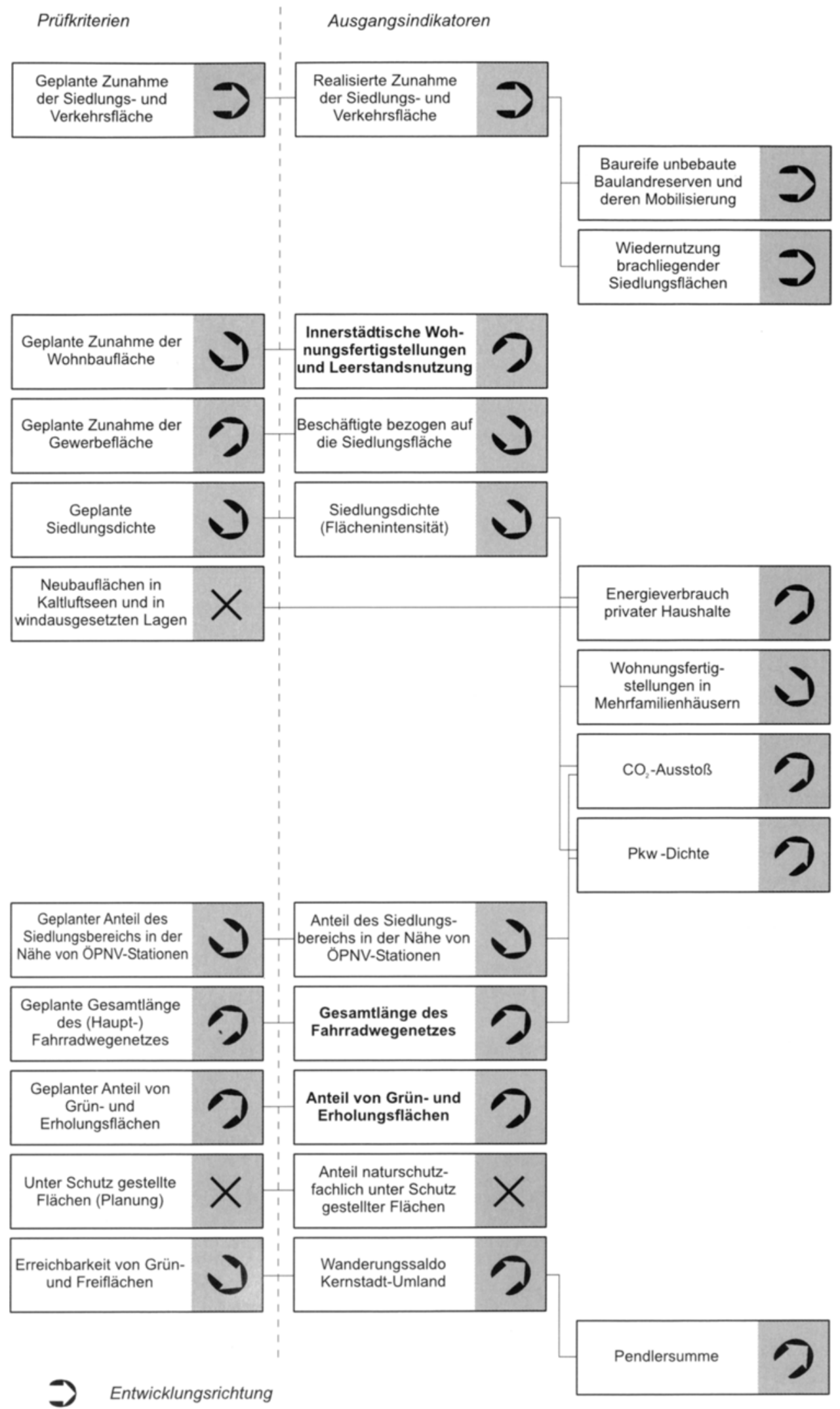

Fettdruck: Gewünschte Entwicklungsrichtung

X nicht erhebbar 


\section{Erfolgsfaktoren für eine indikatorenbasierte Nachhaltigkeitsprüfung}

In der beispielhaften Anwendung der indikatorenbasierten Nachhaltigkeitsprüfung ließen sich vier Erfolgsfaktoren identifizieren, die maßgeblich zu einer erfolgreichen Anwendung des Instruments beitrageñ. Dies sind die Festlegung von Zielwerten, die akteursbezogene Differenzierung von Indikatorensätzen, ein hoher Integrationsgrad der Planung sowie ein kooperatives und transparentes Verfahren.

\subsection{Die Bedeutung von Zielwerten}

In der Indikatorendebatte wird die Bedeutung von Zielwerten betont (Hardi/Zdan 1997, S. 3; Birkmann et al. 1999, S. 19). Dies wird durch die indikatorenbasierte Nachhaltigkeitsprüfung unterstrichen. Mit der Festlegung eines Zielwerts für die einzelnen Indikatoren erhält die Bewertung der Planung eine höhere Aussagekraft, da neben der Entwicklungstendenz eines Indikators dessen Ausgangsniveau in die Bewertung miteinbezogen werden kann. So ist etwa die positive Entwicklung eines Indikators, der bislang sehr weit vom gewünschten Zielwert entfernt ist, höher zu bewerten als die positive Entwicklung eines Indikators, der den gewünschten Zielwert bereits erreicht hat.

Durch Zielwerte erhält man für jeden Indikator eine zweite Bewertungsdimension. Der Umgang mit Zielkonflikten wird erleichtert, da durch das Vorhandensein von Zielwerten ein Maßstab existiert, um bei der Verbesserung des einen Indikators und der gleichzeitigen Verschlechterung des anderen Indikators eine Gewichtung zugunsten des einen oder des anderen Indikators vorzunehmen.

Für die ExWoSt-Indikatoren wurden bislang keine allgemeinen Zielwerte definiert (Kreibich 1999, S. 142). Die indikatorenbasierte Nachhaltigkeitsprüfung für das Heidelberger Modell Räumliche Ordnung musste daher auf den Bezug zu den Zielwerten verzichten. Für weitere Prüfungen nach der dargestellten Methodik ist es zu empfehlen, dass im Zuge der Aufstellung eines Indikatorensatzes das Setzen von Zielwerten durch Politik und Öffentlichkeit berücksichtigt wird. Zielkonflikte lösen sich dadurch nicht auf; der Umgang mit ihnen wird aber erleichtert.

\subsection{Die Bedeutung differenzierter Indikatorensätze}

Indikatorensätze sollen eine überschaubare Anzahl an Indikatoren enthalten, die allgemeinverständlich und anschaulich sind, damit sie für die Öffentlichkeit transportierbar werden (Birkmann 1999, S. 124). Allerdings wird mittlerweile gefordert, Indikatorensätze differenzierter zu gestalten, um sie in den Verwaltungen auch als Planungs- und Steuerungsinstrument einsetzen zu können (Gehrlein 2002, S. 245). Die Prüfkriterien der indikatorenbasierten Nachhaltigkeitsprüfung stellen eine mögliche Form der Ausdifferenzierung dar. Sie gelten für den abgesteckten Bereich der räumlichen Gesamtplanung, ohne dabei den Bezug zum Ausgangsindikatorensatz zu verlieren.

Als Konsequenz wird vorgeschlagen, die Prüfkriterien mittelfristig durch Planungsindikatoren zu ersetzen, die einen lokalen Indikatorensatz ergänzen und spezifizieren. Mehrere Vorteile ergeben sich daraus: Die Ableitung der Prüfkriterien aus den Indikatoren wird durch das einmalige Aufstellen eines Indikatorensatzes für Planungsindikatoren ersetzt, der kontinuierlich und langfristig angewendet wird. Dadurch werden Prüfkriterien mit indirekten Wirkungszusammenhängen entbehrlich, und es stehen Zeitreihen für alle Indikatoren zur Verfügung. Beide Aspekte erhöhen die Aussagekraft der Nachhaltigkeitsprüfung erheblich. Zudem ist die Anwendbarkeit des Indikatorensatzes in der Prüfung garantiert. Für allgemeine Indikatorensätze hingegen ist nicht garantiert, dass sie für die Anwendung auf der Ebene der Flächennutzungsplanung geeignet sind.

\subsection{Die Bedeutung integrierter Planungen}

Ob ein Plan zu einer nachhaltigen Entwicklung beiträgt oder nicht, hängt nicht nur von den inhaltlichen Darstellungen $a b$, sondern in großem Maße auch von den Darstellungsmöglichkeiten selbst. Der Flächennutzungsplan ist grundsätzlich geeignet, um zu einer nachhaltigen Raumentwicklung beizutragen (Apel et al. 2001, S. 223), da durch ihn wichtige Rahmensetzungen vorgenommen werden und kumulative Wirkungen Berücksichtigung finden können. In der Anwendung der indikatorenbasierten Nachhaltigkeitsprüfung wird gleichzeitig deutlich, welche Anforderungen an die Flächennutzungsplanung zu stellen sind, damit sie dieser Aufgabe gerecht werden kann.

Die Umsetzung einer nachhaltigen Raumentwicklung ist eine hoch integrative Aufgabe. Neben Fragen des Boden- und Naturschutzes spielen vor allem Fragen des Verkehrs und der Siedlungsstruktur eine Rolle. Während naturschutzfachliche Fragen durch die Integration der Landschaftsplanung in den meisten Bundesländern im Flächennutzungsplan Berücksichtigung finden, beschränken sich die Aussagen zum Thema Verkehr in den meisten Flächennutzungsplänen auf die Darstellung von Hauptverkehrsstraßen und Bahnanlagen (Bunzel et al. 1994, S. 21). Aus Sicht 
einer nachhaltigen Raumentwicklung ist zu fordern, dass der integrative und überfachliche Charakter der Flächennutzungsplanung durch die Aufnahme von weiteren Darstellungen zum öffentlichen Verkehr und zum Radverkehr gestärkt wird. Dadurch wird dem Umweltverbund in der Gesamtplanung ein höherer Wert beigemessen. Siedlungsstruktur und Verkehr müssen integrativ gedacht werden und als Gesamtpaket bewertbar sein. Das ist effektiver und nachvollziehbarer, als wenn die eine Planung der anderen hinterherhinkt.

Die Ergebnisse der dargestellten indikatorenbasierten Nachhaltigkeitsprüfung haben darüber hinaus unterstrichen, dass neben dem direkten Flächenverbrauch der Siedlungsdichte eine zentrale Bedeutung zukommt. Die Schaffung einer optimalen Siedlungsdichte könnte dadurch unterstützt werden, dass die Flächennutzungsplanung durch Darstellungen zur baulichen Dichte oder Dichtemodelle (vgl. Stadt Heidelberg 1999) ergänzt wird. Durch die Differenzierung der Siedlungsdichte auf Block- oder Quartiersebene lassen sich Planungen zur Umsetzung einer nachhaltigen Raumentwicklung präzisieren. Untersuchungen zu Erreichbarkeiten und zum Energieverbrauch erhalten eine höhere Genauigkeit.

\subsection{Die Bedeutung von Partizipation und Transparenz}

In der dargestellten Form trägt die indikatorenbasierte Nachhaltigkeitsprüfung durch die Art der Informationsbereitstellung zu einem rationalen Abwägungsergebnis bei. Sie kann darüber hinaus die Akzeptanz von Planung erhöhen, wenn sie in ein transparentes und kooperatives Verfahren eingebettet ist. Die indikatorenbasierte Nachhaltigkeitsprüfung setzt damit an den Erkenntnissen kooperativer Planungsprozesse (vgl. Bischoff et al. 2001) und der kooperativen Indikatorenentwicklung an (vgl. Koitka/Kreft 2000). Auch die Auswahl von Indikatoren beinhaltet stets eine Werthaltung der beteiligten Akteure (Birkmann et al. 1999, S. 18 ). Vor diesem Hintergrund wird empfohlen, bereits auf der Stufe der Indikatorenauswahl auf eine breite Beteiligung zu achten.

Die kooperativ entwickelten Indikatoren stellen für die betreffende Gemeinde eine Konkretisierung des allgemeinen Leitbilds Nachhaltigkeit dar. Da sie gleichzeitig die Grundlage für die Durchführung der indikatorenbasierten Nachhaltigkeitsprüfung bilden, werden diese gemeinsam entwickelten Zielvorstellungen in beispielhafter Weise in die Planung aufgenommen. Dadurch ist eine wichtige Forderung kooperativer Planungsverfahren erfüllt. Weiterhin sollte das Ergebnis der Nachhaltigkeitsprüfung der Öffentlichkeit zugäng- lich sein, um die Verwendung der erwähnten Zielvorstellungen transparent zu machen.

Für die Wirkung der indikatorenbasierten Nachhaltigkeitsprüfung ist diese Einbettung ins Aufstellungsverfahren von ebenso großer Bedeutung wie die dargestellte Methodik. An dieser Stelle soll allerdings auf eine ausführliche Betrachtung hierzu verzichtet werden (vgl. eingehender Reuß 2003).

\section{Schlussbetrachtung}

Die indikatorenbasierte Nachhaltigkeitsprüfung trägt zu einer rationalen Abwägung bei, da sie die Operationalisierung der Leitvorstellung der nachhaltigen Raumentwicklung unterstützt. Der Aufwand zur Einführung einer solchen Prüfung ist im Vergleich zu anderen Ansätzen gering, da sie auf bereits häufig vorhandenen Indikatorensätzen aufbauen kann. Zudem liegen die Rohdaten in vielen Fällen vor. Die Intention der indikatorenbasierten Nachhaltigkeitsprüfung liegt damit weniger in der Informationsbeschaffung als in der Informationsaufbereitung. In der Zusammenschau der Indikatoren und der Betrachtung von Zeitreihen werden Entwicklungen und Zusammenhänge leichter erkennbar und transparent. Durch die hohe Anschaulichkeit und Transparenz eignet sich das Konzept in besonderer Weise für kooperative Planungsprozesse.

Eine entscheidende Bedeutung für die Wirksamkeit der Nachhaltigkeitsprüfung kommt der Indikatorenauswahl zu. Die Indikatoren bilden die Bewertungsgrundlage und beeinflussen das Ergebnis der Prüfung entscheidend. Damit die Indikatoren auf eine ausreichende Akzeptanz stoßen, ist daher besonders auf eine sorgfältige und partizipativ unterstützte Auswahl der Indikatoren zu achten. Besonders für den Bereich der Ökonomie liegen bislang nur wenige allgemein anerkannte, aussagekräftige und durch Planung beeinflussbare Nachhaltigkeitsindikatoren vor. Deren Integration in die Nachhaltigkeitsprüfung muss noch verbessert werden.

Für die Zukunft bieten vor allem die Wirkungszusammenhänge zwischen planbezogenen Prüfkriterien und Ausgangsindikatoren ein breites Forschungsfeld. Im Sinne einer stärkeren Ausdifferenzierung von Indikatoren ist anzustreben, auf diese Weise spezielle Planungsindikatoren zu entwickeln, welche die Anwendung der indikatorenbasierten Nachhaltigkeitsprüfung erleichtern und damit zur Etablierung der Nachhaltigkeitsindikatoren als Planungs- und Steuerungsinstrumente beitragen können. 


\section{Literatur}

Apel, D.; Böhme, C.; Meyer, U.; Preisler-Holl, L. (2001): Szenarien und Potentiale einer nachhaltig flächensparenden und landschaftsschonenden Siedlungsentwicklung. Teil 1. - Berlin

Birkmann, J. (1999): Indikatoren für eine nachhaltige Entwick lung. In: Raumforschung und Raumordnung 57, H. 2-3, S. 120 131

Birkmann, J.; Koitka, H.; Kreibich, V.; Lienenkamp, R. (1999): Indikatoren für eine nachhaltige Raumentwicklung. Methoden und Konzepte der Indikatorenforschung. - Dortmund

Bischoff, A.; Selle, K.; Sinning, H. (2001): Informieren, Beteiligen, Kooperieren. Eine Übersicht zu Formen, Verfahren, Methoden und Techniken. 3. Aufl. - Dortmund

Bunzel, A.; Elsner, T.; Lunebach, J. (1994): Flächen sparen in der räumlichen Planung. Ergebnisse einer Untersuchung von Bebauungsplänen, Flächennutzungsplänen und Regionalplänen. - Berlin

Fuhrich, M. (2001): Kompass für den Weg zur Stadt der Zukunft. Indikatorengestützte Erfolgskontrolle nachhaltiger Stadtentwicklung. - Bonn

Gehrlein, U. (2002): Nachhaltigkeitsindikatoren auf kommunaler und regionaler Ebene: bisherige Erfahrungen und Entwicklungsbedarf. Ergebnisse einer bundesweiten Befragung. In: Raumforschung und Raumordnung 60, H. 3-4, S. 239-247

Geier, S.; Holz-Rau,C.; Krafft-Neuhäuser, H. (2001): Randwanderung und Verkehr. In: Internationales Verkehrswesen 53, H. 1+2, S. $22-26$
Hardi, Peter; Zdan, Terrence (1997): Assessing Sustainable Development: Principles in Practice. Winnipeg

Jacoby, C. (2000): Die Strategische Umweltprüfung (SUP) in der Raumplanung. Instrumente, Methoden und Rechtsgrundlagen für die Bewertung von Standortalternativen in der Stadt- und Regionalplanung. - Berlin

Koitka, H., Kreft, H. (2000): Gemeinsam Indikatoren entwickeln! Ein neuer Baustein für erfolgreiche regionale Agenda-21-Prozesse. In: Raumforschung und Raumordnung 58, H. 6, S. 488-498

Kreibich, V. (1999): Entwicklung von Nachhaltigkeitsindikatoren im ExWoSt-Forschungsfeld "Städte der Zukunft“. In: Birkmann, J.; Koitka, H.; Kreibich, V.; Lienenkamp, R. (Hrsg.): Indikatoren für eine nachhaltige Raumentwicklung. Methoden und Konzepte der Indikatorenforschung. Dortmund, S. 128-159

Reuß, A. (2003): Die indikatorenbasierte Nachhaltigkeitsprüfung. In: RaumPlanung, H. 108/109, S. 145-148

Reuß, A. (2002): Indikatorenbasierte Nachhaltigkeitsprüfung auf der Ebene der Flächennutzungsplanung. Dargestellt am Beispiel der Städte Münster und Heidelberg. Diplomarbeit. - Dortmund

Stadt Dortmund (2001): Bevölkerungsbewegung. - Dortmund

Stadt Heidelberg (1999): Siedlungsstrukturkonzept. - Heidelberg

Stadt Heidelberg (2000): Modell Räumliche Ordnung. - Heidelberg

Dipl.-Ing. Albrecht Reuß, SiP

Hans Lamparter GmbH

Bahnhofstraße 4

73235 Weilheim/Teck

E-Mail: albrecht.reuss@hl-ingenieure.de 